\title{
PROPUESTA DE ENFERMERÍA: EMBARAZO EN LA ADOLESCENCIA, APLICACIÓN DEL MODELO DE SISTEMAS.
}

\section{NURSING PROPOSAL: TEENAGE PREGNANCY, APPLICATION OF MODEL SYSTEMS.}

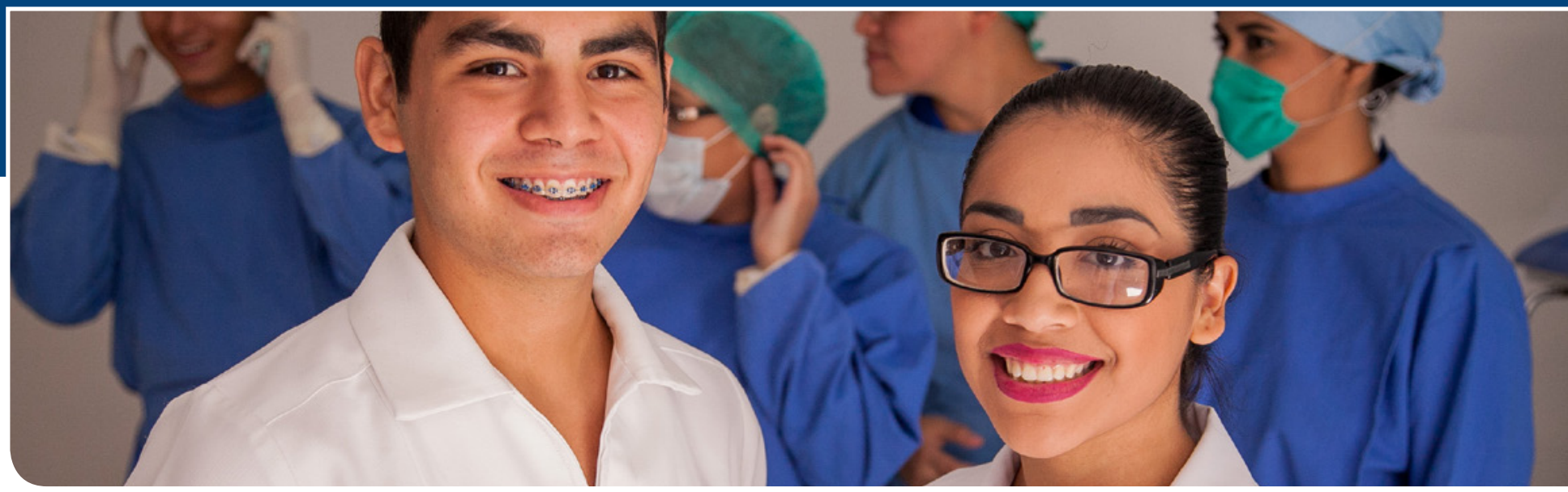

\section{ABSTRACT}

The teenage pregnancy has become a public health problem for the risks posed not only for the adolescent but also for the product and the family, resulting in changes in the structure of society. Therefore for nurses is relevant intervention to be carried out from a viewpoint of the discipline through Betty Neuman Systems Theory, so this proposal will improve the quality through arises the structure and organization of care provided to the adolescent users.

Keywords: Pregnancy, adolescence, nursing.

(*) Maestra en Ciencias de Enfermería Docente de tiempo completo. Departamento de Enfermería. Universidad de Sonora. Email: alejandrafavela@enfermeria.uson.mx

${ }^{(* *)}$ Maestro en Gestión de la Salud y Educación. Técnico Académico. Departamento de Enfermería. Universidad de Sonora. Email: juan.lopez@ enfermeria.uson.mx

\section{RESUMEN:}

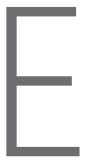

I embarazo en la adolescencia se ha convertido en un problema de salud pública, por los riesgos que representa no solo para la adolescente sino también para el producto y en el núcleo familiar, lo que produce cambios en la estructura de la sociedad. Por lo tanto para el personal de enfermería resulta relevante la intervención que se debe llevar cabo desde un punto de vista de la disciplina a través de la teoría de sistemas de Betty Neuman, en base a lo anterior plantea esta propuesta que permitirá mejorar la calidad a través de la estructura y organización del cuidado que se proporciona a las usuarias adolescentes.

Palabras claves: Embarazo, adolescencia, cuidado de enfermería

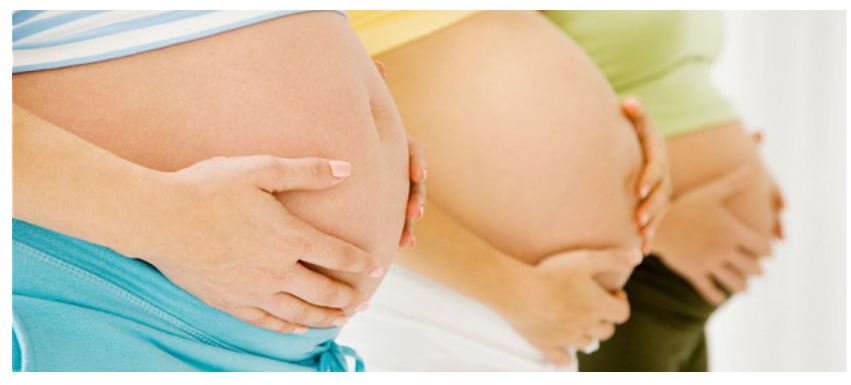

SANUS - DEPARTAMENTO DE ENFERMERÍA 


\section{INTRODUCCIÓN}

La salud sexual y reproductiva de los adolescentes en México, representa uno de los asuntos de mayor relevancia tanto en el ámbito académico como en el político, dado la importancia que tiene este grupo poblacional que actualmente es uno de los más grandes.(1) En México para la atención de los Adolescentes la Secretaria de Salud ha dispuesto la creación de diversos programas, pero pesar de que las acciones de salud reproductiva están dirigidas tanto al hombre como a la mujer, se enfatizan aquellas dirigidas a esta última, justificando este hecho en que es el cuerpo de la mujer donde sucede la fecundación, el embarazo y el parto, siendo ella y el feto o recién nacido los que sufrirán las consecuencias de cualquier complicación en el proceso, incluso la muerte ${ }^{(2-4)}$

El Consejo Nacional de Población (CONAPO) estima que actualmente existen en el país 22.4 millones de mujeres y hombres adolescentes entre los 10 y 19 años de edad. El embarazo en adolescentes cobra cada vez mayor importancia debido a que las mujeres de 15 a 19 años de edad constituyen el grupo de mayor tamaño entre los grupos quinquenales de mujeres en edad fértil. El porcentaje de nacimientos en madres adolescentes aumentó de $15.6 \%$ a $18.7 \%$ entre 2003 y 2012. Sonora se encuentra por encima del promedio nacional de nacimientos de madres adolescentes con 19.8\%, informó el Consejo Estatal de Población

Entre las competencias de Enfermería están ejecutar y evaluar intervenciones destinadas a atender las respuestas humanas dentro del proceso salud-enfermedad en cualquier etapa del ciclo de vida, en este caso en particular, se llevó a cabo una propuesta para abordar la salud reproductiva de la adolescente a través de la organización y coordinación del cuidado de enfermería basada en un modelo de la disciplina, como lo es el modelo de sistemas de Betty Neuman, lo que le permitirá mejorar la calidad en la prestación del cuidado. 


\section{MARCO DE REFERENCIA}

\section{Modelo de Sistemas de Betty Neuman}

EL modelo de sistemas de Betty Neuman está basado en la teoría general de sistemas y refleja la naturaleza de los organismos como sistemas abiertos en interacción entre ellos y con el entorno. ${ }^{(5)}$ Neuman define estrés como la respuesta no especifica del cuerpo a cualquier demanda que se le haga, aumentando la necesidad de ajuste, los elementos estresores pueden ser positivos o negativos.

Asimismo adapta el Modelo de niveles de prevención de Caplan, y relaciona estos niveles de prevención con enfermería. La prevención primaria, protege al organismo antes de que entre en contacto con el estresor. La prevención secundaria busca reducir el efecto de los elementos estresantes a través del diagnóstico precoz y de un tratamiento eficaz de los síntomas de la enfermedad, lo que Neuman describe como el fortalecimiento de las líneas internas de resistencia. La prevención terciaria reduce los efectos residuales de los elementos estresantes y devuelve al cliente el bienestar después del tratamiento.

Considera a la persona como cliente en interacción reciproca con el entorno; este puede ser el individuo, familia, un grupo, la comunidad o un problema social. Un sistema es abierto cuando los elementos intercambian energía de la información en una organización compleja. El entorno son las fuerzas internas y externas que afectan y se ven afectadas por el cliente en cualquier momento. La salud incluye un movimiento continuo pleno del bienestar a la enfermedad. El bienestar óptimo se alcanza cuando se satisfacen todas las necesidades del sistema.

Para Neuman, la Enfermería es una profesión única que se ocupa de todas las variables que afectan la respuesta del individuo frente al estrés, donde la percepción de la enfermera influye en el cuidado que se suministra, por lo tanto se debe valorar la percepción del cliente y del cuidador. A continuación se describe al sistema cliente: la adolescente.

La adolescencia es el período de transición entre la niñez y la vida adulta durante el cual acontece la maduración sexual. La OMS la delimita cronológicamente entre los 10 y 19 años, aunque actualmente diferencia tres períodos, que la extienden hasta los 24 años: pre- adolescencia temprana, de 10 a 14 años, correspondiente a la pubertad; adolescencia media o propiamente dicha, de 14 a 18 años y la adolescencia tardía, de 19 a 24 años. ${ }^{(6,7)}$

Uno de los objetivos específicos del trabajo con la población joven es disminuir los embarazos no deseados, ya que constituyen una condición de riesgo lo cual es una prioridad desde el punto de vista de salud pública. Otra consecuencia es que las jóvenes embarazadas abandonan tempranamente la escuela y con ello restringen sus posibilidades de desarrollo, representando una condición de inequidad de género en la sociedad $(2,4)$

\section{Efectos del embarazo en la adolescente}

Según la OMS, la edad más segura para el embarazo es de los 20 a los 24 años de edad. Algunas de las razones médicas para evitar el embarazo en menores de 18 años es que no se ha completado el desarrollo óseo y no se ha alcanzado la masa mineral máxima, así como la relativa inmadurez del canal del parto. Entre las complicaciones más frecuentes están la anemia, las infecciones bacterianas graves, el parto prematuro, el parto obstruido y prolongado, la desproporción céfalo-pélvica, la muerte fetal y la formación de fístulas recto-vaginales - cistovaginales. La mortalidad relacionada con el embarazo y el parto, es de dos a cinco veces más alta entre las mujeres menores de 18 años de edad que entre las de 20 a 29 años de edad.(8)

El embarazo adolescente, generalmente no planificado y no deseado, 


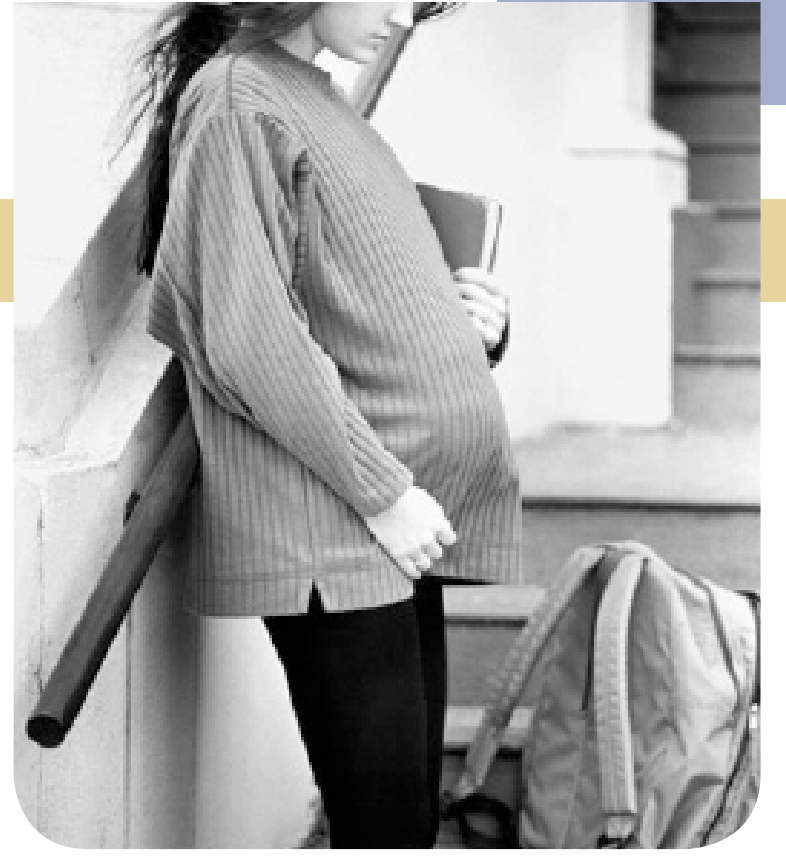

también supone un impacto negativo sobre la condición emocional y económica de la joven, además de condicionar su estilo de vida constituye un problema más social que médico. Una vez tomada la decisión de continuar con el embarazo la joven tiene tres alternativas ${ }^{(9,10)}$

- Maternidad en soltería: Esta alternativa posibilita el desarrollo de las capacidades de la joven pero puede ser objeto de actitudes discriminatorias hacia la joven y su entorno.

- Matrimonio: La joven adolescente que no está preparada para ser madre tampoco está para ser esposa y se va a casar con otro adolescente que tampoco lo está para ser padre y esposo. El nuevo matrimonio socialmente admitido solo representa el agravamiento de los problemas económicos, educativos y de relación afectiva que trajo consigo el embarazo. En nuestro medio un $25 \%$ de los matrimonios jóvenes vuelven a tener otro embarazo en el año siguiente al nacimiento de su primer hijo y un $12.4 \%$ de parejas adolescentes terminan separándose. estos factores determinan el embarazo adolescente y al no corregirse condicionan que más del $50 \%$ de las jóvenes adolescentes que se embarazan sean a su vez, hijas de mujeres que fueron madres adolescentes.

- Adopción: Constituye una alternativa cuando se decide no interrumpir la gestación y es la más compleja porque genera angustia y culpabilidad. Los padres de una adolescente que deja su hijo en adopción presentan una sensación de vergüenza, pena y dolor que suele mitigarse cuando la hija se casa y queda nuevamente embarazada. La adolescente tiene sensaciones similares, siendo bastante frecuente que muchos años después del abandono siga lamentando su decisión y preguntándose cómo estará su hijo.

- Aborto: Las complicaciones del aborto representan la tercera causa de mortalidad materna en el país y se ha señalado que muchas de las muertes maternas atribuidas a la hemorragia del embarazo son en realidad provocadas por complicaciones de abortos inseguros. ${ }^{(7,8,11)}$

\section{Control del Embarazo}

Las jóvenes no buscan apoyo al inicio del embarazo ya que se niegan a admitir la posibilidad de la gestación por lo que retrasan de forma significativa el diagnóstico e inicio de la atención prenatal. Es habitual que el diagnóstico se realice por encima de la semana 20 de gestación. El factor más importante que incide sobre el resultado perinatal no es la edad de la paciente, sino el control de la gestación, siendo éste el factor que reduce sensiblemente las complicaciones de este grupo de gestantes, obteniendo mejores resultados en los embarazos controlados en tres o más ocasiones. ${ }^{(3)}$

Además las jóvenes realizan más abusos dietéticos durante su embarazo, que las madres de mayor edad. Fuman más que las adultas considerándose que la tasa de fumadoras alcanza el $40 \%$, mientras que alrededor del $20 \%$ beben alcohol y el $5 \%$ consumen marihuana u otras drogas no legales.

\section{Riesgo Reproductivo}

El riesgo reproductivo se define como la probabilidad de sufrir daño durante el proceso de reproducción, este es un indicador que nos permite identificar a aquellas mujeres, familias o poblaciones más vulnerables. Los factores de riesgo reproductivo son aquellas características que se presentan en la adolescente que la hacen vulnerable al daño reproductivo, como la presencia de ciertas características biológicas, psíquicas, genéticas, ambientales, sociales, culturales, económicas o políticas que actúan entre sí. $(3,7)$ 
Entre los riesgos preconcepcionales universales que presentan las adolescentes: edad (menores de 18 años), primera gestación, intervalo intergenésico menor a dos años y antecedentes de historia obstétrica complicada. En cuanto al riesgo reproductivo obstétrico, los factores de riesgo son los mismos que en la etapa anterior, con la diferencia que si no se intervino en esa etapa, el riesgo aumenta por la misma gestación. Un problema trascendental en esta etapa es el aborto, el cual aumenta la mortalidad materna si se complica. El riesgo reproductivo perinatal, además de considerar los riesgos maternos (durante el embarazo, el parto y el puerperio) incluye los riesgos para el feto o recién nacido. ${ }^{(2)}$

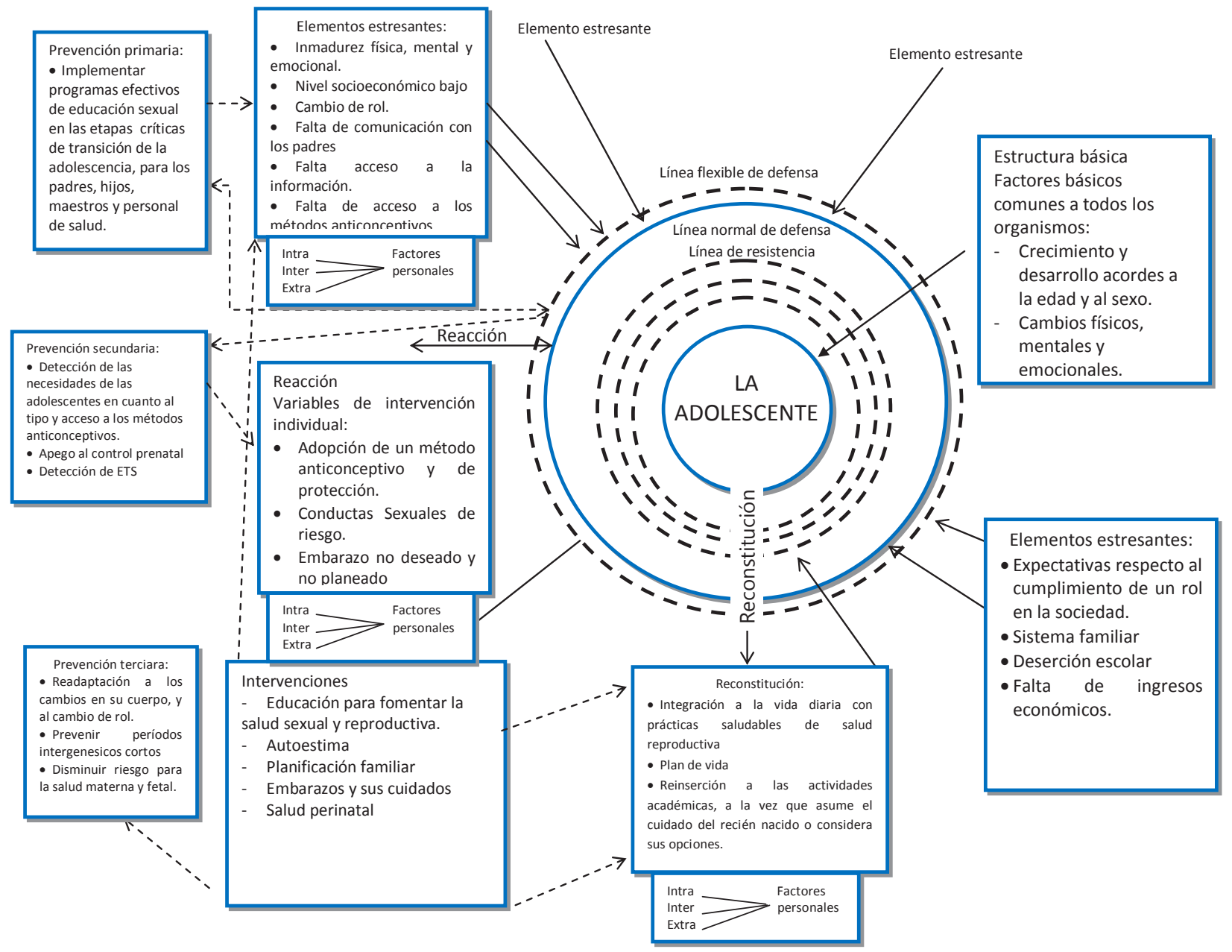

Figura 1. La salud Reproductiva de la adolescente a través del Modelo de Sistemas de Neuman

\section{PROPUESTA DE ENFERMERÍA PARA ABORDAR EL EMBARAZO EN LA ADOLESCENCIA}

La presente propuesta se aborda a través del modelo de sistemas de Betty Neuman el embarazo en la adolescente. Donde el cliente es la adolescente, esta a su vez está inmersa en una dinámica familiar, que tiene en su estructura básica los elementos de crecimiento y desarrollo comunes a todos los adolescentes acordes a su edad y sexo, produciendo cambios físicos, mentales y emocionales (variables fisiológicas, psicológicas, socioculturales, de desarrollo y espirituales). Las líneas de resistencia le ayudaran a sobrepasar estos cambios sin ningún problema superando esta etapa de su vida.

La línea normal de defensa, es el continuo a lo largo del tiempo que le servirán a la enfermera para valorar las desviaciones del bienestar normal del cliente, incluyen su estilo de vida y su estadio de desarrollo. La línea flexible de defensa puede verse alterada rápidamente en un breve período de tiempo, evita que los elementos estresantes crucen la línea normal de defensa y afecten el bienestar del sistema, 


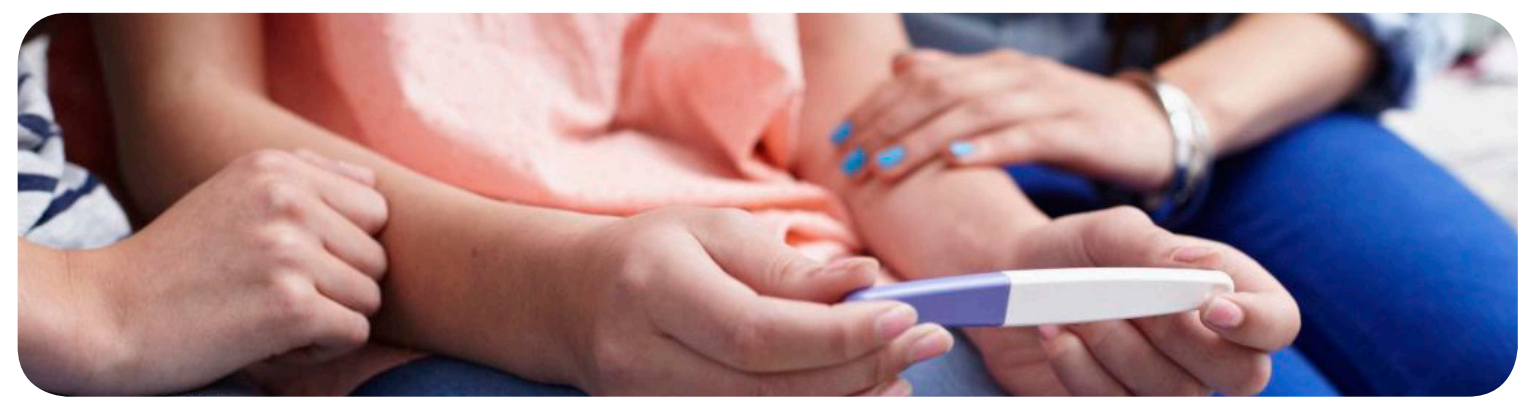

en este caso, la inmadurez, las expectativas que se tengan respecto al rol de la adolescente en la sociedad, la interacción con el sistema familiar, la deserción escolar, la falta de ingresos económicos, algún tipo de enfermedad o cambio de apariencia física la falta de acceso a los métodos anticonceptivos, o a información necesario respecto los cambios que presenta, pueden ser elementos estresantes para la adolescente. La prevención primaria se aplica cuando estos elementos estresantes se identifican o se sospecha que pueden causar una reacción, pero aún no ha tenido lugar.

En el caso de las adolescentes se reconoce que el mayor cambio que se les presenta en cuanto al crecimiento es en el aspecto sexual, por los procesos fisiológicos que se están produciendo, situación en la que requieren tener información confiable, generalmente a las primeras personas que recurren son los maestros, los amigos o los padres de familia, por lo que hay que hacerles llegar a todas estas personas que rodean a las adolescentes los programas de educación sexual. Determinar las necesidades de aprendizaje, el nivel educativo y la calidad de la información que se requiere manejar en estos programas educativos para que sean efectivos, y beneficien al cliente en su transición por la adolescencia.

El grado de reacción representa la inestabilidad del sistema que tiene lugar cuando los elementos estresantes invaden la línea normal de defensa. En el caso de la adolescente que requiere información y no la ha recibido podremos observar que presenta conductas sexuales de riesgo o un embarazo no deseado o no planeado o al contrario puede mostrar apego por un método anticonceptivo. En la prevención secundaria observamos, la detección de necesidades de las adolescentes en cuanto al tipo y acceso a los métodos anticonceptivos, detección de enfermedades de transmisión sexual o en su caso de un embarazo, si ya está embarazada revisar cómo lleva su control prenatal.

En la prevención terciaria se pretende conseguir que la cliente recupere su estabilidad óptima, y poder retroceder hacia la prevención primaria. En este caso, educación para fomentar la salud sexual y reproductiva, fomentar a la autoestima, planificación familiar, el embarazo y sus cuidados, salud perinatal. La reconstitución tiene lugar después del tratamiento de las reacciones de los elementos estresantes, integración a la vida diaria llevando a cabo prácticas saludables de salud reproductiva, retomar su plan de vida, reinsertar o continuar con las actividades académicas a la adolescente, a la vez que asume el cuidado del recién nacido o considera sus opciones, como la adopción.

\section{CONCLUSIONES}

El modelo de sistemas de Betty Neuman es utilizado para describir las respuestas físicas del cuerpo al estrés y el proceso de adaptación al mismo. En el caso de la adolescente, todo el proceso de transición que está atravesando le genera un estado de estrés constante el cual puede alterar su equilibrio en la dinámica del proceso salud enfermedad. Este proceso está compuesto por tres funciones básicas: alarma, resistencia y agotamiento; la primera se desencadena cuando se percibe el estrés, mismo que preparará al organismo para defenderse; pero si el estrés persiste, la fase de resistencia no se hará esperar y el cuerpo moviliza todas las capacidades de afrontamiento y todos los mecanismos de defensa del individuo para alcanzar la homeostasis, si la situación estresante ha sido abrumadora por su larga duración, los recursos del individuo se agotan y el organismo comienza a debilitarse y de persistir se puede producir la muerte

El ejercicio de la sexualidad en edades tempranas puede generar diversos efectos negativos como los ya antes mencionados La complejidad de esta problemática requiere una transformación reflexiva por parte de los adultos que educan adolescentes, implica que estos actores desarrollen la capacidad de generar satisfactores para comprender la sexualidad de los adolescentes de modo que estos últimos puedan ejercer una sexualidad sana, responsable y libre. 
La maternidad no deseada obstaculiza el ejercicio de los derechos humanos básicos de las mujeres y representa una violación a distintos acuerdos internacionales firmados y ratificados por México; asimismo, representa una violación al Artículo $4^{\circ}$ de la Constitución Mexicana, en el cual se establece que todas las personas tienen el derecho a decidir de manera libre, responsable e informad sobre el número y el espaciamiento de sus hijos.

Los programas de salud sexual y reproductiva deben orientarse hacia la satisfacción de las necesidades de las adolescentes, generando, por medio de la educación y la consejería, los siguientes satisfactores:

- La capacidad de disfrutar de una vida sexual satisfactoria y sin riesgos responde a la necesidad de afecto.

- La posibilidad de ejercer el derecho a procrear o no y la capacidad para decidir el número y espaciamiento de los hijos son satisfactores que responden a la necesidad de libertad.

- El derecho a obtener información le proporciona al individuo elementos para tomar decisiones libres e informadas y sin sufrir discriminación, coerción ni violencia; responde a las necesidades de entendimiento y de libertad.

- El acceso y la posibilidad de elección de métodos de regulación de la fecundidad satisfacen necesidades de protección y de libertad.

- La eliminación de la violencia doméstica y sexual y el derecho a recibir adecuados servicios de atención a la salud independientemente del sexo, la edad, la etnia, la clase, la orientación sexual o el estado civil de la persona, teniendo en cuenta sus necesidades específicas de acuerdo con su ciclo vital, satisfacen las necesidades de protección.

Sin duda México es uno de los países que cuentan con normatividades y programas de salud para sus ciudadanos en todo el ciclo de vida, sin embargo se observa la incongruencia de que la información y los recursos no están llegando a quien los requiere, puesto que el número de embarazos no deseados o no planificados en las adolescentes no disminuye significativamente, ni tampoco el índice de muertes maternas. Además, la mayoría de los adolescentes que tienen relaciones sexuales por primera vez refieren no utilizar métodos de protección.

Como parte de la disciplina de enfermería, sugiere realizar investigaciones con las adolescentes de diversas edades, para saber si son los mismos estresores en la adolescencia temprana o tardía, y así poder llevar a cabo las intervenciones. La salud de reproductiva, en particular la de la adolescente, requiere especial atención, ya que se convirtió en un problema de salud pública, además de las consecuencias que tiene para esa futura mujer adulta, en todos sus sistemas, individual, familiar y comunitario.

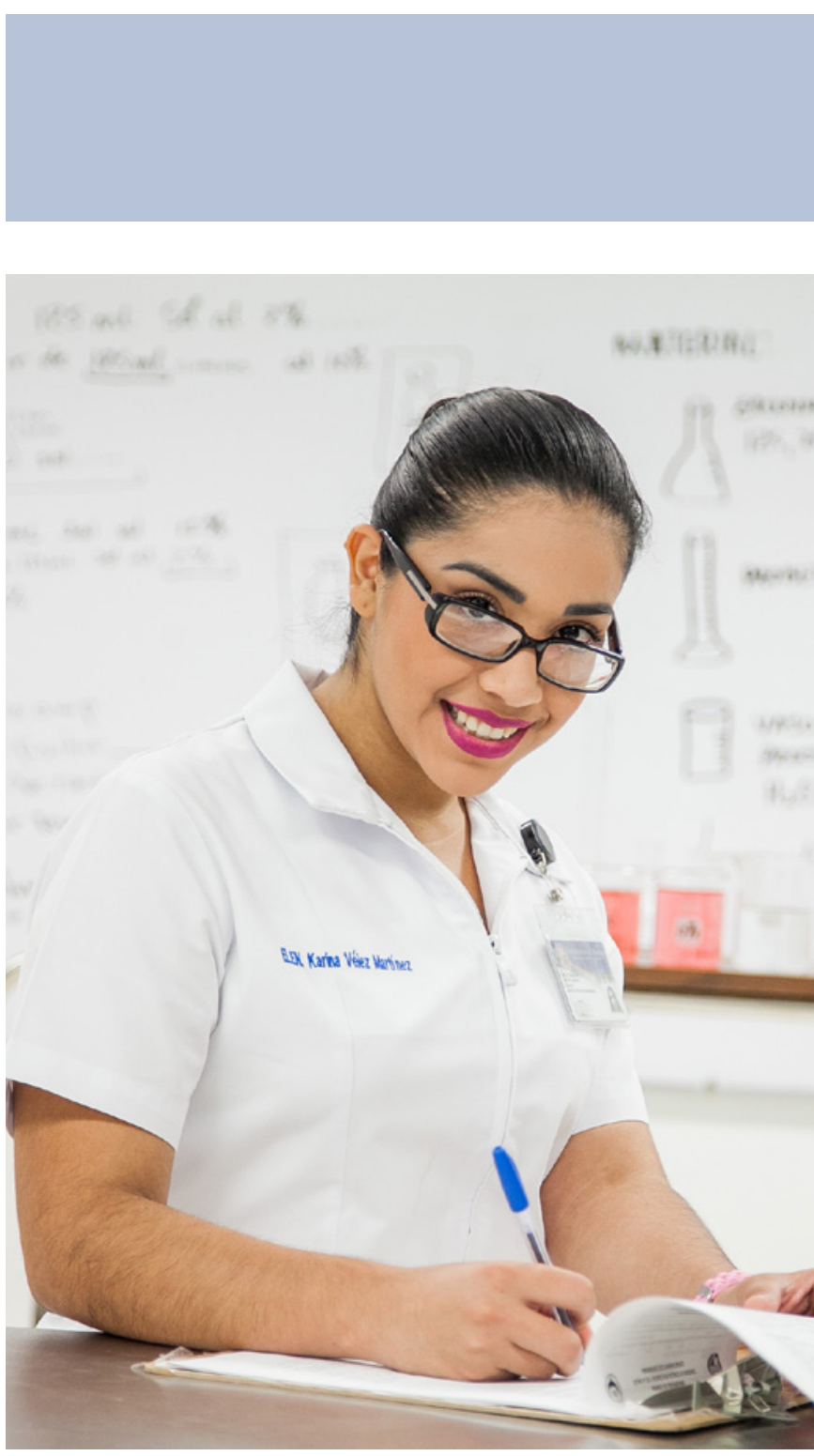




\section{REFERENCIAS BIBLIOGRÁFICAS}

1. Juárez F, Gayet C. Salud sexual y reproductiva de los adolescentes en México: un nuevo marco de análisis para la evaluación y diseño de políticas: Universidad Autónoma del Estado de México; 2005.

2. Gutiérrez ID. Salud reproductiva: Concepto e importancia: Proyecto Salud Reproductiva MPSSP-UNFPA-OPS/OMS; 1992.

3. Gil JAA, Hernández BM. La salud sexual y reproductiva en la adolescencia: un derecho a conquistar : manual educativo para prestadores de servicios de salud: CELSAM; 2001.

4. Allen-Leigh B, Villalobos-Hernández A, Hernández-Serrato MI, Suárez L, Vara Edl, de Castro $F$, et al. Inicio de vida sexual, uso de anticonceptivos y planificación familiar en mujeres adolescentes y adultas en México. salud pública de méxico. 2013;55:S235-S40.

5. Alligood MR, Marriner-Tomey A. Modelos y teorías en enfermería: Elsevier; 2011.
6. Loundgren R. Protocolos de investigación para el estudio de la salud sexual y reproductiva de los adolescentes y jóvenes varones en América Latina. OPS/FNUAP/Kellogg/ASDI; 2000.

7. Rice FP, Salinas MEO. Desarrollo humano: estudio del ciclo vital: Prentice-Hall Hispanoamericana; 1997.

8. Ros R, Morandi T, Cozzetti E, Lewintal C, Cornella J, Surís J. Manual de Salud Reproductiva en la Adolescencia. España: Sociedad Española Contracepción (SEC). 2001.

9. Manual de salud reproductiva en la adolescencia: aspectos básicos y clínicos: Sociedad Española de Contracepción; 2001.

10. Towle MA. Asistencia de enfermería maternoneonatal: Pearson Educación; 2010.

11. Carrillo MA. Reflexiones Finiseculares: Universidad Autónoma Metropolitana; 2000.

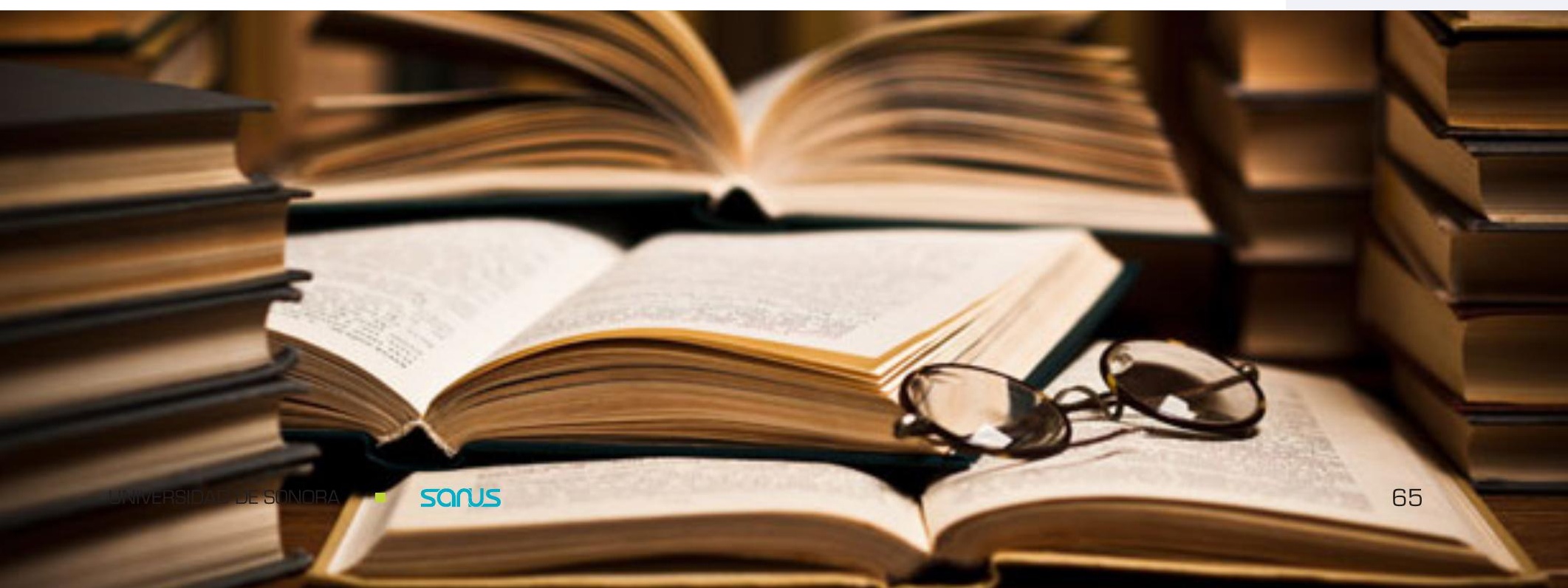

